\title{
Characterization of Fennel Flavors by Solid Phase Trapping-Solvent Extraction and Gas Chromatography-Mass Spectrometry
}

\author{
Yeon-Jae Shin, ${ }^{\text {a }}$ Mi-Jin Jung, Nam-Sun Kim, ${ }^{\text {c }}$ Kun Kim, and Dong-Sun Lee" \\ Department of Chemistry, Seoul Women 's University, Seonl 139-774, Korea. "E-mail: dslee@swu.ac.kr \\ Received June 29, 2007
}

\begin{abstract}
IIeadspace solid phase trapping solvent extraction (IIS-SPTE) and GC-MS was applied for the characterization of volatile flavors from fennel, anise seed, star-anise, dill seed, fennel bean, and Ricard aperitif liquor. Tenax was used for IIS-SPTE adsorption material. Recoveries, precision, linear dynamic ranges, and the limit of detection in the analytical method were validated. There were some similarities and distinct differences between fennel-like samples. The Korean and the Chinese fennels contained trans-anethole, $(+)$-limonene, anisealdehyde, methyl chavicol as major components. The volatile aroma components from star anise were characterised by rich trans-anethole, (+)-limonene, methyl chavicol, and anisaldehyde. $\Lambda$ dditionally, principal component analysis (PC $\Lambda$ ) has been used for characterizing or classifying eight different fennel-like samples according to origin or other features. $\Lambda$ quite different pattern of dill seed was found due to the presence of apiol (dill).
\end{abstract}

Key Words : Headspace solid phase trapping solvent extraction (HS-SPTE), GC-MS, Fennel, Anethole

\section{Introduction}

Fennel (Foeniculum vulgore Miller) is biennial or perennial herb of Umbelliferae up to 2 meters high, with feathery leaves and golden yellow flowers. The fruit (Foeniculi Fructus, Korean; so-hoe-hyang) of fennel is a dry seed from 4-9 mm long, half as wide or less, and grooved. Dried fennel seed is an aromatic, anise-flavored spice, brown or green in color, they slowly turn a dull gray as the seed ages.

Fennel seed is used as a flavoring for foods and beverages, and the essential oil from the seed and plant is used in condiments, creams, soaps, perfumes, cosmetic, and pharmaceuticals. Fennel fruits are aromatic, stimulant, and carminative, analgesic, anti-inflammatory, antispasmodic, diuretic, emmenagogue, expectorant, galactogogue, hallucinogenic, laxative, stimulant and stomachic. Fennel can relieve intestinal gas accumulations and gastro intestinal spasm..$^{1.2}$

Fennel seeds are sometimes confused with the anise seed (Anisi Fructus, Pimpinello omisum), which is very similar in taste and appearance, though smaller. In this respect, its use resembled that of anise and dill (Anethi Fructus, Anethum sowa or Anethom groneolens). In Asia, anise is less known, as fennel and star anise (Ilicinom vertm) being more easily available and more popular. Fennel and fennel oil are listed in the Korean Pharmacopoeia (KP VIII-2), whereas aniseed (Pimpinella anisum), anise oil (Ilicium verum or Pimpinella anisum), and powdered aniseed are listed in the British Pharmacopoeia. Anise may substitute fennel in Northern Indian recipes, but it is a less suited substitute for star anise in Chinese foods. In China, fennel is found in the wellknown five-spice powder, along with star anise and cinnam-

\footnotetext{
"Present address: SCINCO CO., LTD., Seoul 135-010, Korea

'Present address: GRACEL DISPLAY INC., Seoul 133-833, Korea

'Present address: Korea Food \& Drug Administration, Seoul 122-704, Korea
}

on. China and Vietnam are major producers of transanethole from the star anise, and are supplying a large proportion of the demand.

Volatile compounds of fennel seeds extracted by simultaneous distillation-extraction (SDE) ${ }^{3}$ and supercritical fluid extraction (SFE) showed similar compositions, with transanethole, estragole, and fenchone as the main components. ${ }^{+}$ All available methods in the analytical scale can be applied to the extraction of flavors of plant origin. However, isolation techniques by either steam distillation or solvent extraction have some shortcomings, namely losses of thermolabile compounds, low extraction efficiency, long extraction time, degradation of compounds and toxic solvent residue. To overcome these problems several approaches have been used to extract volatile flavor compounds of plant origin. Altemative to conventional techniques, supercritical fluid extraction, subcritical water extraction at temperatures between $100^{\circ} \mathrm{C}$ and $374^{\circ} \mathrm{C}$ and a pressure high enough to maintain the liquid state, and head space solid-phase microextraction (HS-SPME) are also reported. ${ }^{5-11}$

The purpose of the present study is to develop a simple, rapid, sensitive and reproducible procedure for the quantitative determination of flavor compounds in the fennel and fennel-like samples. The method in the present paper for the extraction and determination of flavor compounds in fennel and fennel-like samples is based on the headspace solidphase trapping solvent extraction (HS-SPTE) and gas chromatography-mass spectrometry (GC/MS) ${ }^{6.7}$ Additionally, principal component analysis (PCA) has been used for characterizing or classifying eight different fennel-like samples according to origin or other features. ${ }^{12-14}$ This approach could be employed in pattem recognition problems and chemotyping to distinguish fennel seed, anise seed, star anise and dill seed, because these samples have similar patterns of flavor compositions. 


\section{Experimental}

Materials and reagents. Eight fennel-like samples illustrated in Figure 1 were used in this study. Two different kinds of fennel (Foeniculam vilgare) samples were purchased from the local markets of Suncheon in Korea and Wuhan in China. The dried fruit samples of anise seed (Pimpinella anisim) and dill seed (Anetheum sowa) were purchased from McCormick (Chicago, USA). Fennel bean (Hui Xiang Dou) is green bean steamed with fennel and salt, and purchased from the Xian Heng Jiu Dian (Shaoxing, Zejiang, China). Star anise (Illicium verum) harvested in Guang-Xi of China was purchased from the local market of Wuhan (China) and fried star anise was commercial product from the Shanghai MeiFeng Food (China). Aperitif anise liquor (Ricard ${ }^{\text {s. }}$ ) was $45 \%$ alcoholic liquor of anise seed and purchased from La Fee Verte (Marseille, France).

All working reference standards were of analytical grade and were purchased from Sigma-Aldrich (St. Louis, MO, USA) and Tokyo Kasei (Nihonbashi, Tokyo, Japan). Organic solvents of chromatographic grade were purchased from Sigma-Aldrich.

Headspace solid phase trapping solvent extraction (HS-SPTE). Flavor compounds were collected from the fenne]-like samples by using a HS-SPTE apparatus illustrated in Figure 2 designed in our laboratory. ${ }^{\text {1.16 }}$ Each sample (about $3 \mathrm{~g}$ ) was filled in a $20 \mathrm{~mL}$ round flask, but different amounts were used for anise seed, star anise and fried star anise $(50 \mathrm{mg}$ each) and Aperitif anise liquor $(10$ $\mathrm{mL}$ ) were used, respectively. Volatile flavor components were collected for $60 \mathrm{~min}$ at ambient temperature by use of the Pasteur pipet $(0.565 \mathrm{~cm}$ i.d. $\times 15 \mathrm{~cm}$ length, Kimia Pajooh Co. Ltd., Tehran, Iran) as a trap-housing which is packed with about $260 \mathrm{mg}$ of 2,6-diphenylene-oxide polymer resin adsorbent (Tenax, particle size 250-177 $\mu \mathrm{m}, \mathrm{P} / \mathrm{N}$ 1-1982, Supelco, Bellefonte, PA, USA) and glass wool plugs, according to the procedure identical with that reported in our previous papers. ${ }^{16}$ Prior to use, the Tenax trap was

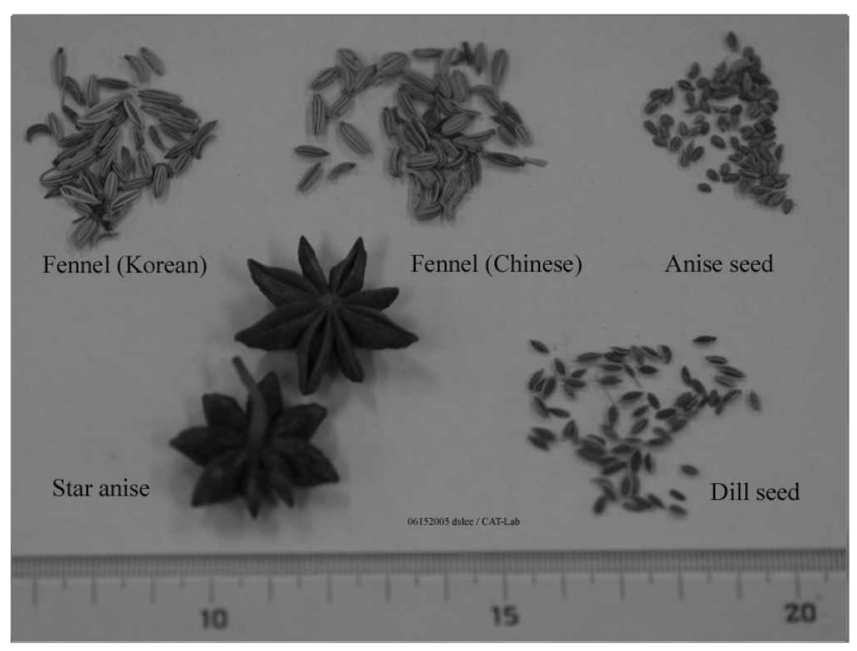

Figure 1. Photographs of fennel seed, anise seed, star anise, and dill seed.

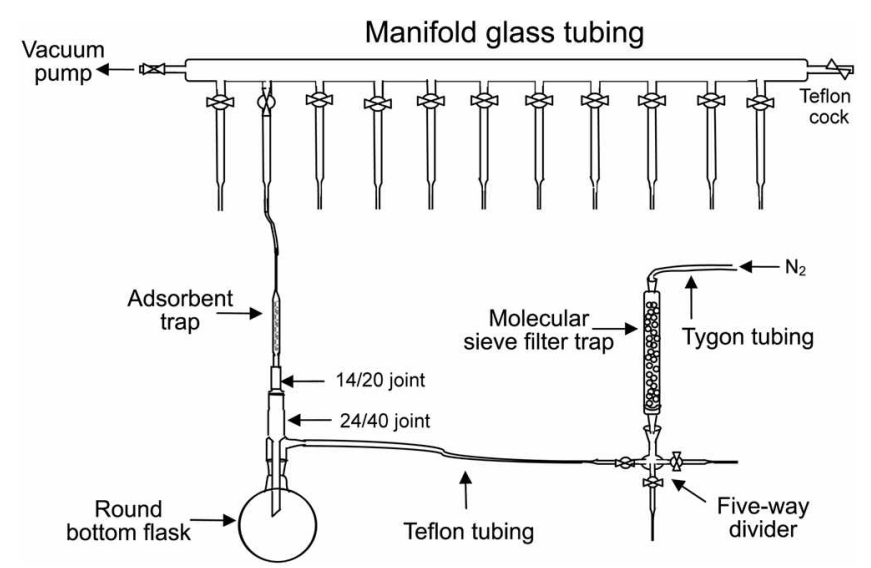

Figure 2. Scheme of solid-phase tapping-solvent extraction (SPTE) apparatus.

pre-rinsed with $2 \mathrm{~mL}$ of petroleum ether to remove impurities. The inlet of the Pasteur pipet was attached to the flask $(20 \mathrm{~mL})$ containing the fennel or fennel-like samples. An oil-free electric vacuum pump (Vacuubrand, Wertheim, Germany; diaphragm ME2 model, $2.4 \mathrm{~m}^{3} / \mathrm{h}$ ) and a polytetrafluoroethylene (PTFE, Teflon) valve restrictor were connected with Tygon tubing to the outlet end of the trap via glassmanifold. A purified nitrogen gas $(99.99 \%)$ flow ca. 400 $\mathrm{mL} / \mathrm{min}$ was passed into a flask and out through the absorbent trap under reduced pressure. After a run, the retained compounds were eluted by three extractions with $2 \mathrm{~mL}$ of petroleum ether. The elute was then further concentrated to a final volume of approximately $200 \mu \mathrm{L}$ on water bath at $35^{\circ} \mathrm{C}$ by using a Kudema-Danish type device jointed with a Snyder column. Aliquots were analyzed by GC/MS.

Gas chromatography-mass spectrometry (GC/MS). GC/MS analyses were carried out by using a Trace GC 2000 and a $\mathrm{GC}-\mathrm{Q}$ plus jon trap $\mathrm{MS}^{\mathrm{n}}$ (Thermoquest-Finnigan, Austin, TX, USA) with electron impact ionization mode. Chromatographic separations were performed on a fused silica cross-linked 6\% cyanopropylphenyl-94\%-dimethylsiloxane copolymer capillary column (DB-624, $30 \mathrm{~m}$ length $\times 0.25 \mathrm{~mm}$ i.d. $\times 1.4 \mathrm{\mu m}$ film thickness, temperature limits $-20 \sim 260^{\circ} \mathrm{C}, \mathrm{J} \& \mathrm{~W}$ Scientific, Folsom, CA, USA). Flow rate of carrier gas $(\mathrm{He}, 99.9995 \%$ ) was $1.0 \mathrm{~mL} / \mathrm{min}$. The injector temperature was $240^{\circ} \mathrm{C}$. A split injection with a ratio of 1:30 was used. The injected sample volume was 1 iA. The oven temperature program was $50^{\circ} \mathrm{C}(3 \mathrm{~min})-5^{\circ} \mathrm{C}$ $\mathrm{min}-220^{\circ} \mathrm{C}(5 \mathrm{~min})$. Transfer line temperature was $230^{\circ} \mathrm{C}$. The electron impact ionization mass spectrometer was operated as follows; ionization voltage, $70 \mathrm{eV}$; ion source temperature, $200^{\circ} \mathrm{C}$. The measuring mode was scanned from 50 to 350 mass ranges. The identity of volatile compounds was assigned by comparison of their Kovats retention indices (I), relative to the homologous series of $n$ alkane $\left(\mathrm{C}_{8}-\mathrm{C}_{20}\right)$ and comparison of the obtained mass spectra of relevant chromatographic peaks with those of authentic standards and with corresponding spectra of the NIST and Wiley libraries.

Analysis of enantiomers from fennel sample. HP 5890 
series II GC (Hewlett-Packard, Avondale, PA, USA) with flame ionization detector (FID) and a 30\% heptakis (2,3-diO-methyl-6-O- $t$-butyldimethyl-silyl)- $\beta$-cyclodextrin column (Cyclosil-B, J\&W, $30 \mathrm{~m} \times 0.25 \mathrm{~mm} \times 0.25 \mathrm{um}$ film thickness) were used to analyze enantiomers from fenmel-like samples. The oven temperature program was $50^{\circ} \mathrm{C}(3 \mathrm{~min})$ $5^{\circ} \mathrm{C} / \mathrm{min}-220^{\circ} \mathrm{C}(5 \mathrm{~min})$; injector, $240^{\circ} \mathrm{C}$; detector, $250^{\circ} \mathrm{C}$; split ratio, $1: 30$; carrier gas flow rate of $\mathrm{N}_{\star} 99.99 \%, 1 \mathrm{~mL} /$ $\mathrm{min}$; air, $300 \mathrm{~mL} / \mathrm{min}$; hydrogen, $30 \mathrm{~mL} / \mathrm{min}$.

Principal component analysis (PCA). PCA was accomplished with multivariate statistical analysis program (MVSAP, version 4.0 ) software developed in our laboratory and prevalidated by using known values and data sets. ${ }^{12.14}$ From a multivariate data matrix having $p$ variables and $n$ samples, principal component scores were computed by using MVSAP.

\section{Results and Discussion}

Identification of the chemical constituents in the fennel like samples by gas chromatography and mass spectrometry. The total ion chromatograms (TIC) on a DB-624 column of the aroma components collected from fennel analyzed by HS-SPTE and GC-MS are shown in Figure 3.

Table 1 gives a list of 10 aroma components found for eight fennel-like samples. The retention indices as well as characteristic mass spectral ions of each peak are also given. The peak numbers in Figure 3 correspond to the numbers indicated in the first column of Table 1 , in increasing order of retention time.

Comparison of aroma components found in fennels with those found in anise seed, star anise, dill seed, fennel bean, fried star anise, and Aperitif anise liquor is summarized in Table 2. Among the flavor compounds identified, transanethole was the primary component with relative percentile composition (normalized peak area \%) of $78.07 \%-88.58 \%$ for fenmel, $81.55 \%$ for anise seed, $95.71 \%$ for star anise, $88.76 \%$ for fried star anise, and $94.12 \%$ for Aperitif anise liquor. Fennel bean has similar flavor composition with fennels. Star anise and fried star anise contained transanethole, $(+)$-limonene, anisealdehyde, methyl chavicol,
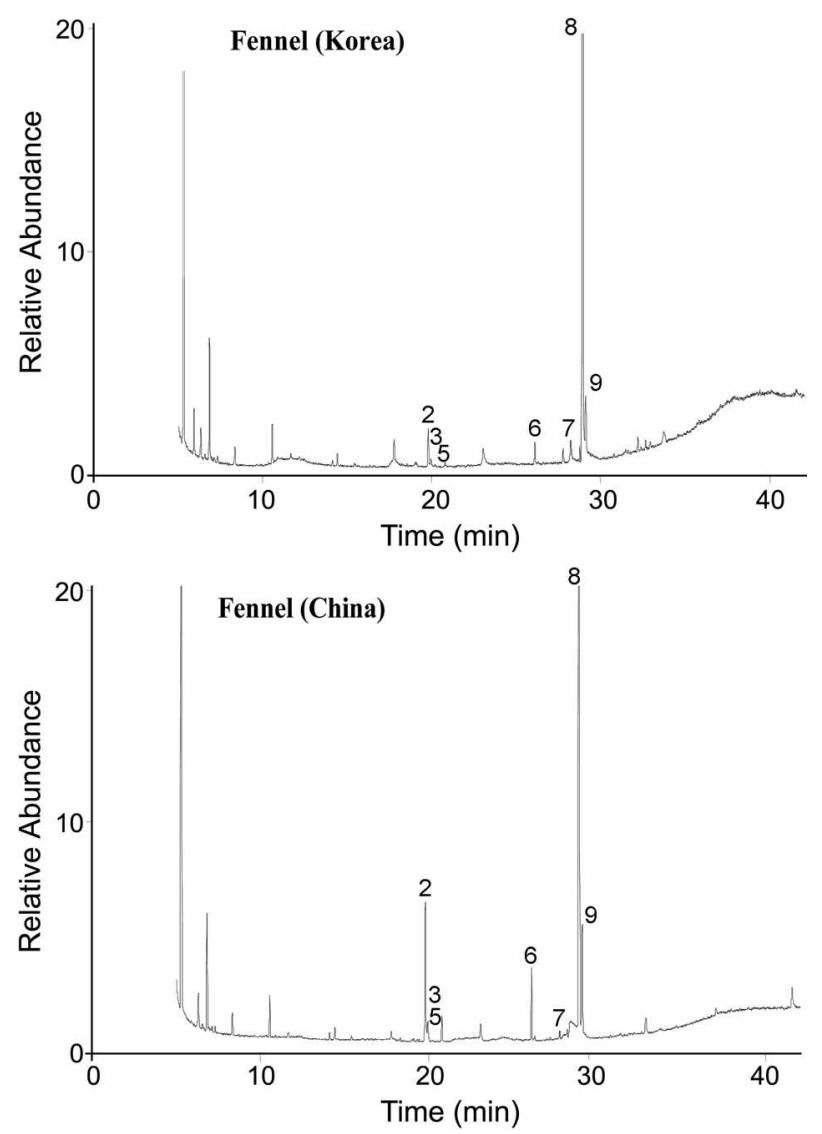

Figure 3. Total ion chromatograms of flavors in fennel samples by SPTE-GC/MS.

carvone, phellandrene, $\gamma$-terpinene, and $\beta$-myrcene, whereas apiole (Dill), carvone and $(+)$-limonene were major components of dill seed investigated.

The enantiomeric analyses of limonene and carvone were undertaken using a cyclosil-B column with HS-SPTE and GC-FID. The enantiomer of limonene in the flavor emitted from Korean fennel seed was found in the $(+)$ form. Chiral compounds from natural origins usually exist as one predominant optical isomer. Enantiomers show different odor properties. $\left(^{+}\right)$-limonene was found to have an orange odor,

Table 1. Characteristic mass spectral ions of volatile flavor compounds identified from fennel-like samples

\begin{tabular}{llccccc}
\hline $\begin{array}{l}\text { Peak } \\
\text { No. }\end{array}$ & Compound & $\begin{array}{c}t_{\mathrm{R}} \\
\text { (min) }\end{array}$ & $I$ & $\mathrm{Mr}$ & $\begin{array}{c}\text { Base peak } \\
\mathrm{m} / \mathrm{z}\end{array}$ & \multicolumn{1}{c}{$\begin{array}{c}\text { Characteristic mass spectral ions (EI) } \\
\mathrm{m} / \mathrm{z} \text { (relative abundance \%) }\end{array}$} \\
\hline 1 & $\beta$-Myrcene & 18.20 & 1002 & 136 & 91 & $53(27), 69(30), 77(53), 91(100), 93(90), 107(5), 121(5), 136(7)$ \\
2 & (+)-Limonene & 19.88 & 1050 & 136 & 67 & $53(25), 67(100), 79(48), 93(46), 107(20), 121(17), 136(16)$ \\
3 & $p$-Cymene & 19.96 & 1052 & 134 & 119 & $65(4), 91(50), 119(100), 134(30)$ \\
4 & $\alpha$-Phellandrene & 20.01 & 1054 & 136 & 91 & $51(5), 65(15), 77(65), 91(100), 93(60), 119(3), 136(15)$ \\
5 & $\gamma$-Terpinene & 20.79 & 1076 & 136 & 91 & $50(15), 65(10), 77(50), 91(100), 93(65), 105(10), 121(15), 136(20)$ \\
6 & Methyl chavicol & 26.10 & 1245 & 148 & 148 & $50(40), 63(30), 77(45), 91(38), 105(32), 117(47), 113(25), 147(60), 148(100)$ \\
7 & Carvone & 28.22 & 1317 & 150 & 93 & $54(50), 65(10), 79(45), 91(80), 93(100), 108(60), 135(20), 151(50)$ \\
8 & trans-Anethole & 28.98 & 1345 & 148 & 148 & $51(11), 63(8), 77(24), 91(30), 115(22), 121(17), 133(12), 147(50), 148(100)$ \\
9 & Anisaldehyde & 29.10 & 1349 & 136 & 135 & $50(15), 63(20), 77(50), 92(12), 107(10), 135(100), 136(50)$ \\
10 & Apiole (dill) & 37.96 & 1686 & 222 & 222 & $51(11), 63(10), 77(20), 98(13), 106(13), 121(22), 149(30), 150(10), 177(50)$, \\
& & & & & & $191(22), 207(40), 222(100)$ \\
\hline
\end{tabular}

$I=$ retention indices, $\mathrm{Mr}=$ relative mass 
Table 2. Flavor compositions of fennel-like samples identificd by SPTE and GC-MS

\begin{tabular}{llcccc}
\hline Peak No. & Compound & Fennel (Korea) & Fennel (China) & Anise seed & Star anise \\
\hline 1 & F-Myrcene & - & - & - & $0.04 \pm 4.24$ \\
2 & (+)-Limonene & $1.46 \pm 6.30$ & $6.17 \pm 1.17$ & - & $0.81 \pm 2.00$ \\
3 & $p$-Cymene & $0.41 \pm 5.70$ & $0.69 \pm 3.82$ & - & - \\
4 & $\alpha$-Phellandrene & - & - & - & $0.07 \pm 6.32$ \\
5 & $\gamma$-Terpinene & $0.95 \pm 8.86$ & $1.01 \pm 8.21$ & - & $0.02 \pm 6.03$ \\
6 & Methyl chavicol & $0.81 \pm 6.10$ & $2.98 \pm 0.08$ & $0.97 \pm 1.08$ & $0.31 \pm 5.55$ \\
7 & Carvone & $1.23 \pm 5.16$ & $2.27 \pm 5.05$ & - & $0.12 \pm 8.00$ \\
8 & trans-Anethole & $88.58 \pm 1.07$ & $78.07 \pm 1.74$ & $81.55 \pm 0.01$ & $95.71 \pm 0.01$ \\
9 & Anisaldehyde & $2.87 \pm 8.95$ & $4.78 \pm 1.36$ & $12.10 \pm 0.21$ & $0.44 \pm 3.20$ \\
10 & Apiole (Dill) & - & - & - & - \\
\hline Peak No. & Compound & Dill seed & Fennel bean & Fried star anise & Aperitif Anise liquor \\
\hline 1 & F-Myrcene & - & - & $0.16 \pm 1.00$ & - \\
2 & (+)-Limonene & $10.45 \pm 3.80$ & $20.83 \pm 1.08$ & $3.83 \pm 4.20$ & - \\
3 & $p$-Cymene & - & $2.21 \pm 1.98$ & - & - \\
4 & $\alpha$-Phellandrene & - & - & $1.83 \pm 3.02$ & - \\
5 & $\gamma$-Terpinene & - & $0.84 \pm 4.63$ & $0.37 \pm 0.06$ & - \\
6 & Methyl chavicol & $2.34 \pm 1.70$ & $0.63 \pm 5.69$ & $0.41 \pm 0.51$ & $0.78 \pm 8.97$ \\
7 & Carvone & $24.26 \pm 0.79$ & - & - & - \\
8 & trans-Anethole & $4.92 \pm 2.44$ & $11.51 \pm 0.07$ & $88.76 \pm 0.08$ & $94.12 \pm 0.14$ \\
9 & Anisaldehyde & $0.97 \pm 4.25$ & $2.33 \pm 3.61$ & $2.44 \pm 0.09$ & $3.32 \pm 1.95$ \\
10 & Apiole (Dill) & $39.20 \pm 0.97$ & - & - & - \\
\hline
\end{tabular}

unit: notmalized peak area $(\%) \pm R S D,-:$ Nol detected

while (-)-limonene was found of terpentine type. ${ }^{17}$ However, carvone enantiomers were not resolved under the given conditions.

Validation of the analytical method of HS-STPE and GC/MS. The accuracy of the analytical procedure was assessed by the absolute recovery study. A pair of test samples was prepared for analysis as follows: The first test sample is prepared by adding known amount of authentic standard to a known amount of sample specimen that contains the sought for analyte. The second one is the same known amount of unspiked sample specimen. Both of the test samples were analyzed by the experimental procedure. Triplicate measurements were made on all test samples. Percentile recovery was calculated by comparison of added and found amounts of analyte in spiked samples using the following equation.

$$
\% \text { Recovery }=\left[\left(A_{\circ} \mid s-A_{0}\right) / A_{s}\right] \times 100
$$

where $A_{0 t s}$ is peak area for the sample matrix spiked with known amount of analyte; $A_{0}$ is peak area for the analyte of the unspiked sample matrix; $A_{s}$ is peak area for the known amount (same as the spiked amount) of authentic analyte standard.

The absolute recovery test of three major compounds was carried out. As shown in Table 3, about 45\%-53\% recoveries were obtained for trans-anethole, methyl chavicol, and anisaldehyde. This result means that no exhaustive extraction of three compounds could be obtained by HS-SPTE, because the headspace extraction is a technique of partial extraction.

The precision of the measurements was evaluated using
Table 3 Recoverics of SPTE-GC/MS for three major flavor compounds from spiked anise seeds $(n=3)$

\begin{tabular}{lcc}
\hline Compound & Amount spiked (ng) & Recovery ( \pm RSD) \\
\hline trans-Anethole & 50 & $45.95 \%( \pm 4.39)$ \\
Methyl chavicol & 10 & $45.33 \%( \pm 17.38)$ \\
Anisaldehyde & 10 & $53.42 \%( \pm 8.32)$ \\
\hline
\end{tabular}

the results obtained by repeated analysis of samples. The reproducibility of the SPTE-GC/MS for four major compounds as the relative standard deviation (RSD) of the mean amount and accuracy (\%) is reported in Table 4. RSDs $(n=$ 3 ) range from 1.23 to 4.37 and accuracy (\%) ranges from 1.55 to 7.30 for the mean amount.

Standard calibration curves for limonene, methyl chavicol, trans-anethole, and anisaldehyde are given in Table 5. As represented in Table 4 and 5, a good linearity peak area $v s$. concentration was observed in between picogram and nanogram scale, depending on the compound. This is lower than those observed by Shukui et al. in the microgram scale. ${ }^{18}$ Despite this, the correlation coefficients $\left(R^{2}\right)$ value exceeds 0.97 , and hence appears to be a good linearity.

At high concentrations, the curves deviate from linearity. Since analytical measurements must be made using the linear part of the curve, such linear dynamic ranges are suitable for the analysis of fennel-like sample without dilution.

The limit of detection (LOD) values based on a signal-tonoise ratio $(\mathrm{S} / \mathrm{N}$ ) of 3 were very low, ranging from $0.11 \mathrm{pg} /$ $\mu \mathrm{L}$ for anisaldehyde to $1.09 \mathrm{pg} / \mu \mathrm{L}$ for methyl chavicol (Table 5).

Application to quantitative analyses of real samples. 
Table 4. Reproducibility of the SPTE-GC/MS for four major compounds

\begin{tabular}{|c|c|c|c|c|c|c|}
\hline \multirow{2}{*}{ Compounds } & \multirow{2}{*}{$\begin{array}{l}\text { Real concentration } \\
\qquad(\mu \mathrm{g} / \mathrm{mL})\end{array}$} & \multirow{2}{*}{ Running \# } & \multicolumn{4}{|c|}{ Amount } \\
\hline & & & Found $(u g / \mathrm{mL})$ & Mean $(u g / m L)$ & RSD $(\%)$ & Accuracy $(\%)$ \\
\hline \multirow[t]{3}{*}{ (+)-Limonene } & 40 & 1 & 38.75 & 39.38 & 1.48 & 1.55 \\
\hline & & 2 & 39.91 & & & \\
\hline & & 3 & 39.49 & & & \\
\hline \multirow[t]{3}{*}{ Methyl chavicol } & 20 & 1 & 21.60 & 21.44 & 1.23 & 7.20 \\
\hline & & 2 & 21.58 & & & \\
\hline & & 3 & 21.13 & & & \\
\hline \multirow[t]{3}{*}{ trans-Anethole } & 50 & 1 & 54.94 & 53.65 & 2.77 & 7.30 \\
\hline & & 2 & 52.02 & & & \\
\hline & & 3 & 53.98 & & & \\
\hline \multirow[t]{3}{*}{ Anisaldehyde } & 15 & 1 & 16.09 & 15.45 & 4.37 & 3.00 \\
\hline & & 2 & 14.74 & & & \\
\hline & & 3 & 15.52 & & & \\
\hline
\end{tabular}

Table 5. The limit of detection, dynamic range, and response factor for the determination of five major volatile flavor compounds in fennellike samples using SPTE-GC/MS

\begin{tabular}{lcccl}
\hline Compounds & LOD $(\mathrm{pg} /, \mathrm{L}$ ) & Dynamic range & RF (Area/ng) & Eq. of calibration curve $\left(\mathrm{R}^{2}\right)$ \\
\hline (+)-Limonene & 0.77 & $0.77 \mathrm{pg}-80 \mathrm{ng}$ & 0.046 & $\mathrm{y}=0.0461 \chi-0.1006(0.9980)$ \\
Methyl Carvicol & 1.09 & $1.09 \mathrm{pg}-30 \mathrm{ng}$ & 0.088 & $\mathrm{y}=0.0884 \chi-0.122(0.9888)$ \\
Carvone & 0.69 & $0.69 \mathrm{pg}-90 \mathrm{ng}$ & 0.018 & $\mathrm{y}=0.1825 \chi-0.631(0.9901)$ \\
trans-Anethol & 0.59 & $0.59 \mathrm{pg}-150 \mathrm{ng}$ & 0.089 & $\mathrm{y}=0.0888 \chi-0.2395(0.9919)$ \\
Anisaldehyde & 0.11 & $0.11 \mathrm{pg}-40 \mathrm{ng}$ & 0.045 & $\mathrm{y}=0.0448 \chi-0.225(0.9769)$ \\
\hline
\end{tabular}

The external method has been employed to detemine the major flavor compounds in fennel-like samples. Quantitative data obtained by extemal calibration method is summarized in Table 6 . There are some similarity and distinct differences among fennel-like samples. The Korean and the Chinese fennels contained trans-anethole, limonene, anisaldehyde, and methyl chavicol (estragole or iso-anethole). The volatile aroma component from star anise investigated was characterized by rich trans-anethole, limonene, anisaldehyde, carvone, and methyl chavicol. Several other plants emanate aroma similar to that of anise (Pimpinella anisum). To a lesser extent, dill (Anetheum sowa or Anethwm groveolens) also resemble anise, although their anise fragrance is not so much pure as the former mentioned plants are.

It can be seen that the volatile aroma composition of Aperitif anise liquor showed quite similar compositional pattern with anise seed. There are many applications of anise-flavored liquors in different Mediterranean countries: Pastis in France, Rakl in Turkey, and Ouzo in Greece. In many cases, oil of anise is substituted by oil of star anise in these products, at least partially.

The amounts of secondary compounds like volatile aroma compounds are affected by genetic and geographical factors, climate, soil, and cultivation techniques. Other studies ${ }^{19}$ have demonstrated, using steam distillation techniques, the presence of trans-anethole as the major compound of the essential oils of fennel and anise seed. Anise seed and star anise contain $1 \%-4 \%$ and about $5 \%$ volatile oil, respectively. According to the recent research by Gurbuz, ${ }^{20}$ essential oil levels of Turkish anise varied from $1.3 \%$ to $3.7 \%$ and its major component was trans-anethole ranging from $78.63 \%$ to $95.21 \%$. Oil of anise or star anise has carminative and expectorant properties, but its major component (anethole) has been reported to be the cause of dermatitis in some people. Anethole has two isomers (trans and cis) with the cis isomer being 15-38 times more toxic to animals than the trans one, depending on species. The trans-anethole is more stable than the cis isomer. In natural resources, trans form is the major component, but only the trace amount of the cis isomer is present. ${ }^{21}$ Anethole was formerly considered as an active estrogenic agent of the essential oil of anise. However, further research suggests that the active estrogenic compounds are polymers of anethole, such as dianethole and photoanethole. ${ }^{2}$

The pharmacologic effects of trans-anethole most often noted are reduction in motor activity, lowering of body temperature and hypnotic, analgesic, and anticonvulsant effects. trans-Anethole is largely used as a substrate for the synthesis of various phannaceutical substances such as chloral, an anticonvulsant agent, diethyl stielbesterol and phenobarbital. The antioxidant activity of star anise oil is due to the high percentage of trans-anethole. ${ }^{22}$ In addition, trans-anethole possesses muscle relaxant effect. ${ }^{23}$ Anethole was evaluated for acceptable daily intake (ADI) by the joint FAO/WHO Expert Committee on Food Additives (FAO, 1968 ) in 1967 and given a conditional ADI of $0-1.25 \mathrm{mg} / \mathrm{kg}$ body weight.

At low concentrations, trans-anethole loses its licorice flavor and gives a sweet flavor. Therefore, very small amount of trans-anethole is used in many types of toothpaste to 
Table 6. Quantitation of five inalor flavor compounds from fennel-like samples ( $n=3$ )

\begin{tabular}{|c|c|c|c|c|c|}
\hline Sample & $\begin{array}{c}(+) \text {-Limonene } \\
(\mathrm{ng} / \mathrm{g})\end{array}$ & $\begin{array}{l}\text { Methyl chavicol } \\
\text { (ng/g) }\end{array}$ & $\begin{array}{c}\text { Carvone } \\
(\mathrm{ng} / \mathrm{g})\end{array}$ & $\begin{array}{c}\text { trans-Anethole } \\
(\mathrm{ng} / \mathrm{g})\end{array}$ & $\begin{array}{c}\text { Anisaldehyde } \\
\text { (ng/g) }\end{array}$ \\
\hline Fennel Suncheon, Korea & $1.26 \pm 2.21$ & $0.62 \pm 3.08$ & $2.34 \pm 0.67$ & $18.79 \pm 2.39$ & $2.46 \pm 1.78$ \\
\hline Fennel Wuhan, China & $5.20 \pm 0.40$ & $1.60 \pm 1.32$ & $2.11 \pm 2.27$ & $30.73 \pm 5.12$ & $4.99 \pm 3.77$ \\
\hline Anise seed McCormick, USA & - & $6.32 \pm 1.07$ & - & $1.82\left(: 10^{2}\right) \pm 3.36$ & $1.64(\times 10) \pm 2.89$ \\
\hline Star anise Wuhan, China & $1.41\left(\therefore 10^{3}\right) \pm 1.79$ & $2.70\left(\therefore 10^{2}\right) \pm 1.36$ & $6.67 \pm 4.23$ & $1.61\left(\because 10^{+}\right) \pm 0.91$ & $1.54\left(\div 10^{3}\right\} \pm 4.81$ \\
\hline Dill seed McCormick, USA & $3.14 \pm 3.57$ & $0.83 \pm 2.11$ & $15.26 \pm 1.26$ & $4.33 \pm 6.14$ & $1.64 \pm 4.00$ \\
\hline Fennel bean Xianheng, Shaoxing, China & $1.88 \pm 2.98$ & $0.59 \pm 1.15$ & - & $1.75 \pm 2.09$ & $1.40 \pm 0.08$ \\
\hline Fried star anise Shanghai MeiFeng Food, China & $1.04(\times 10) \pm 1.09$ & $7.25 \pm 4.10$ & - & $7.43\left(\div 10^{2}\right) \pm 4.72$ & $2.86(\times 10) \pm 1.05$ \\
\hline Aperitif anise liquor RICARD ${ }^{*}$, Marseille France & - & $0.19 \pm 0.72$ & - & $1.80\left(\times 10^{2}\right) \pm 1.12$ & $3.15 \pm 6.25$ \\
\hline
\end{tabular}

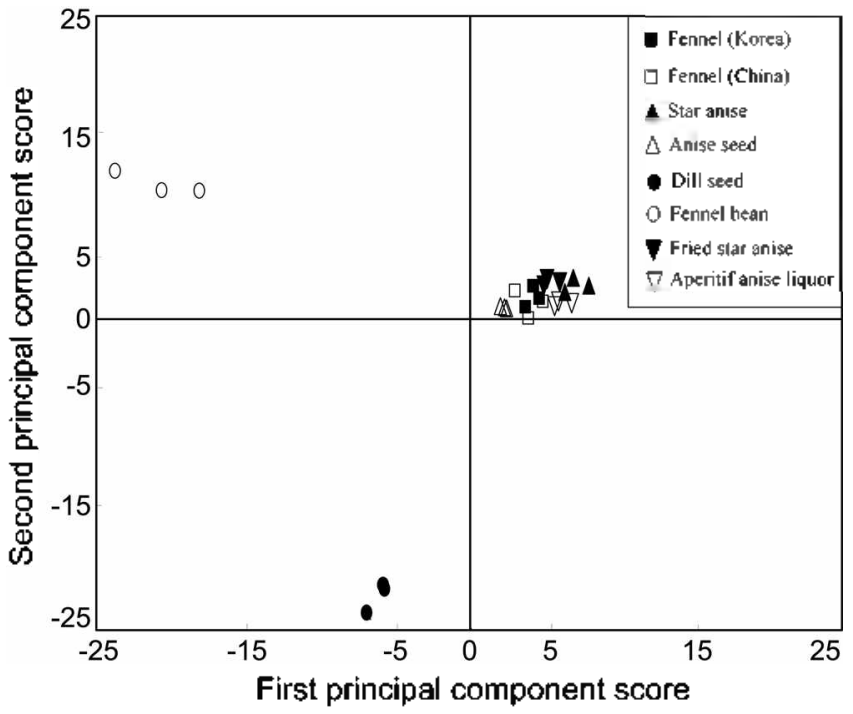

Figure 4. The PC $\Lambda$ plot based on the nonnalized peak area $(\%)$ for flavor compositions of fennel-like samples by STTE and GC-MS.

make them sweet without adding sugar. ${ }^{2+}$

Minor components as well as major components found from botanical materials may be typical for some varieties and provoke a distinction of chemotypes or aroma characteristics. For example, in dill seed, four chemotypes are known, based on the presence of myristicin, or apiole (Dill), or both, or none of them. ${ }^{25}$ Indian dill seed (Anethum sowa) contains dillapiole, while European dill seed (Anethw" graveolens) dose not contain apiole (Dill). According to Bailer et al., ${ }^{26}$ limonene and carvone are the major constituents of European dill seed as well as caraway essential oils. Same authors suggested that carvone/limonene ratio of the dill seed oil mirrors, with astonishing fidelity, the variations of the essential oil contents. Carvone has potential uses for inhibiting the growth of bacteria and some fungi, and for an insect repellent. The most important technical application of carvone is the use as a reversible suppressant of sprouting in stored potatoes and flower bulbs.

Dill seeds contain volatile oil with concentration varying from $1.2 \%$ to $7.7 \%$ according to geographical origin and seasons. The quality of dill oil can fluctuate greatly, depending on the percentage of seed oil, and the physiological maturity of the seeds used for oil. The time of harvest may also be significant, because carvone is synthesized during the day from phellandrene breakdown. Dill herb oil is sometimes adulterated with terpenes from other sources. Both myristicin and apiole (dill) present in dill oil are effective natural insecticides. Myristicin is also known to be responsible for psychoactive and hallucinogenic properties in some other plants, and the content of apiole may be responsible for diuretic properties.

Flavors pattern recognition by principal component analysis. PCA was employed to provide an overview of capacity to distinguish fennel-like flavors based on GC/MS data sets as tabulated in Table 2 and 6. The plots in Figure 4 illustrated the first two principal components scores as a dimension reduction device. A PCA plot showed some important features of the data set such as a significantly different pattern and the presence of characteristic compounds in a sample. As shown in Figure 4 and Table 2, a quite different pattern of dill seed is found due to the presence of apiole (dill). Dill seed containing a different flavor composition can be easily distinguished from other fennel-like samples by the PCA plot. The star anise can be distinguished from other samples based on the very rich trans-anethole (Table 6). These results suggest that PCA is very useful for many aspects of spice flavor industry, including pattem recognition or primary evaluation of category similarity, detection of adulterants, and quality control.

\section{Conclusion}

Headspace-solid phase trapping solvent extraction (HSSPTE) is an effective analytical method for the volatile flavor compounds from fennel, anise seed, star-anise, dill seed, fennel bean, and Ricard aperitif liquor. It has the good linear dynamic range $\left(\mathrm{R}^{2}>0.97\right)$, which was observed in between pictogram and nanogram scale, and low detection limit (LOD $<1.09 \mathrm{pg} / \mu \mathrm{L}$ ). In addition, principal component analysis (PCA) was useful for the characterization or classification of fennel-like samples according to origin or other features. There are some similarity and distinct differences among fennel-like samples.

Acknowledgements. This work was financially supported by Seoul R\&BD Program (2005-10632). 


\section{References}

1. Chandler, R. F.; Hawkes, D. Can. Pharm. J. 1984, /17,28.

2. Albert-Puleo, M. J. Ethopharmacol. 1980, 2, 337.

3. Dietmar, B.; Thomas, B.; Armin, M. J. Agric. Food Chem, 1997 , 45,4554 .

4. Diaz-Maroto, M. C.; Diaz-Maroto Hidalgo, I. J.; SanchezPalomo, E.; Perez-Coello, M. S. J. Agric. Food Chem. 2005, 53, 5385.

5. Lee, D. S. Korean J. Odor Research Engineering 2002, 1, 16.

6. Kim, H. J.; Kim, K.; Kim, N. S.; Lee, D. S. J. Chromatogr: $A$ $2000,902,389$

7. Kim, N. S.; Lee, D. S. J. Chromatogr $A$ 2002, 982, 31.

8. Moura, L. S.; Carvalho, R. N.; Stefanini, M. B.; Ming, L. C.; Meireles, M. A. A. J. Supercrit. Fhtids 2005.,35. 212.

9. Damjanović, B.; Lepojević, Ž.; Živković, V.; Tolic, A. Food Chem. $2005,92,143$.

10. Gámiz-Gracia, L.; Luque de Castro, M. D. Talanta 2000, 51, 1179.

II. Lado, C.; Farkas, E.; Then, M.; Szentmihalyi, K. Proccedings of the $I^{\text {th }}$ Sympositim on Analyical and Emiromental Problems; Galvacs, Z., Ed.; Institute of materials and environmental chemistry chemical research center, Hungarian Academy of Sciences: Szeged, 2004; p 178-182.

12. Yoo, Z. W, Kim, N. S.; Lee, D. S. Bulletin Korean Chem. Soc. 2004, 25,271.
13. Lee, D. S.; Noh, B. S.; Bae, S. Y.; Kim, K. Anal, Chim, Acta 1998 , $358,163$.

14. Lee, D. S.; Lee, E. S.; Kim, H. J.; Kim, S. O.; Kim, K. Antal. Chin. Acta 2001, 429, 323 .

15. Lee, D. S.; Kim, N. S. Bull. Korean Chem. Soc. 2002, 23, 1647.

16. Lee, S. N.; Kim, N. S.; Lee, D. S. Anal. Bioanal. Chem, 2003 377,749 .

17. Shukui, Z; Xin, L.; Li, D.; Jun, X.; Xueli, S.; Hongwei, K.; Guowang. X.; Caiying, W. J. Chromatogr. A 2005, J086, 107.

18. Brenna, E.; Fuganti, C.; Serra, S. Tetrahedron: Asymmetry 2003, 14,1 .

19. Santos, P. M.; Figueiredo, A. C.; Oliveira, M. M.; Barroso, J. G.; Pedro, L. G; Deans, \$. G; Younus, A. K. M.; Scheffer, J. J. C. Phtochen. 1998, 48, 455 .

20. Arslan. N.; Gurbuz. B.; Sarihan, E. O.; Gumuficu. A. Tirk $J$ Agric. For: 2004, 28, 173 .

21. Clark, G S. Perfum. Flavorist 1993, 18,1 I.

22. Besharati-Seidani, A.; Jabbari, A.; Yamini, Y, Anal. Chm Acta $2005,530,155$.

23. Gülçin, I.; Oktay, M.; Kireçcl, E.; Küfrevioğlu, Ö. I. Food Chent. 2003,83,371.

24. Yoshioka, M.; Tamada, T. Biogenic Amines 2005, 19,89.

25. Kruger, H.; Hammer, K. J. Essential Oil Res. 1996, 8, 205.

26. Bailer, J.; Aichinger, T.; Hackl, G; Hueber, K.; Dachler, M. Industiral Crops Products 2001, 14, 229. 